\title{
Controlling the size of thiolated organosilica nanoparticles
}

Article

Accepted Version

Al Mahrooqi, J. H., Mun, E. A., Williams, A. C. and Khutoryanskiy, V. V. (2018) Controlling the size of thiolated organosilica nanoparticles. Langmuir, 34 (28). pp. 8347-8354. ISSN 0743-7463 doi:

https://doi.org/10.1021/acs.langmuir.8b01556 Available at https://centaur.reading.ac.uk/77788/

It is advisable to refer to the publisher's version if you intend to cite from the work. See Guidance on citing.

To link to this article DOI: http://dx.doi.org/10.1021/acs.langmuir.8b01556

Publisher: American Chemical Society

All outputs in CentAUR are protected by Intellectual Property Rights law, including copyright law. Copyright and IPR is retained by the creators or other copyright holders. Terms and conditions for use of this material are defined in the End User Agreement.

\section{www.reading.ac.uk/centaur}

\section{CentAUR}

Central Archive at the University of Reading 
Reading's research outputs online 


\title{
Controlling the size of thiolated organosilica
}

\section{nanoparticles}

\author{
Jamila H. Al Mahrooqi, Ellina A. Mun, Adrian C. Williams, Vitaliy V. Khutoryanskiy*
}

Reading School of Pharmacy, University of Reading, Reading, RG6 6AD, United Kingdom. *Author for correspondence: Tel.: +44(0)118 378 6119. Fax: +44(0)118 378 4644. E-mail: v.khutoryanskiy@reading.ac.uk

\begin{abstract}
:
Nanoparticle characteristics, including their size, are governed by the reagents employed and the reaction parameters. Here, we systemically vary the catalyst, oxygen content, temperature and solvent to modify the size and zeta potential of thiolated organosilica nanoparticles. Particles were synthesized by self-condensation of 3-mercaptopropyltrimethoxysilane (MPTS) in a range of organic solvents in contact with oxygen, with $\mathrm{NaOH}$ and other catalysts. Particle size increased with increasing reaction temperature $\left(70 \pm 1 \mathrm{~nm}\right.$ at $50{ }^{\circ} \mathrm{C} ; 50 \pm 1 \mathrm{~nm}$ at room temperature) but decreased when air was bubbled through the reaction mixture compared to no bubbling. Significant decrease in particle size was seen with increasing the dielectric constant of the solvent and with increasing catalyst concentration; from these we provide empirical equations that can be used to design particle sizes by manipulating the dielectric constant of the solvent (or co-solvents) or by varying $\mathrm{NaOH}$ catalyst concentration when DMSO is the selected solvent.
\end{abstract}


KEYWORDS: silica; nanoparticles; thiols; disulfide bridges; 3-mercaptopropyltrimethoxysilane 


\section{INTRODUCTION:}

The introduction of nanoparticles has led to major advances in fields including targeted therapeutics, medical diagnostics, molecular biology and cell biology. Nanoparticles have been evaluated for cancer cell imaging, ultrasensitive single bacterium detection, DNA microarray detection, and gene delivery [1-4]. To fully exploit nanoparticles in these areas, they should provide strong signals (such as fluorescence), have high specificity and binding affinities and, ideally, should be suitable for use in controlled release systems [3, 4]. Doxil, a $80-85 \mathrm{~nm}$ PEGylated liposome encapsulating doxorubicin hydrochloride and Abraxane with paclitaxel bound to albumin in particles $~ 130 \mathrm{~nm}$, are examples of nano-scale anticancer therapeutics currently approved by the US Food and Drug Administration [6].

Silica nanoparticles are good candidates for biomedical applications since their production and separation are simple, they are hydrophilic, and may be modified or labelled [3]. Further, many biological studies have shown them to be biocompatible and non-toxic [7]. The introduction of functional groups such as amines, thiols and carboxyls is relatively straightforward due to the high surface area and silanol surface concentration, in addition to the highly interconnected open spaces in their three-dimensional structure $[8,9]$. Researchers have sought to define the factors controlling the particle size and morphology of silica nanoparticles. Regulating hydrolysis during synthesis when alkoxysilanes are used as a pure silica source is an effective way to control size and can be achieved by adding alcohol, varying the concentration of the catalyst and by selection of alkoxysilanes of different nature [10]. The temperature, $\mathrm{pH}$, type of catalyst used also affect the size of silica nanoparticles produced [3, 10-14]. 
Thiolated organosilica nanoparticles have attracted considerable attention for their ability to bind transition metals, with opportunities for diverse functionalisation and use in catalysis[5].

Organosilicon molecules containing thiol groups have been successfully used for the synthesis of silsesquioxane- based thermally stable networks [15] and ionic liquids [16]. Thiolated organosilica nanoparticles are typically synthesized by co-condensing 3mercaptopropyltrimethoxysilane (MPTS) with tetraethoxysilane or tetramethoxysilane in protic solvents such as water or alcohols. Co-condensation is induced by the addition of $\mathrm{NaOH}$ or $\mathrm{NH}_{4} \mathrm{OH}$, and the formed material had particle sizes larger than $500 \mathrm{~nm}[3,5]$. Particle size varied by changing the mercaptosilane derivative and concentration [1,2]. $100 \mathrm{~nm}$ fluorescent thiolorganosilica nanoparticles were used for time-lapse fluorescence imaging of mouse peritoneal macrophages which clearly demonstrated cellular uptake and uptake kinetics [17]. Sub-100 nm thiolated organosilica nanoparticles were obtained by self-condensation of MPTS in dimethylsulfoxide (DMSO) in contact with atmospheric oxygen [5, 18]. The resulting nanoparticles were versatile as they can be fluorescently labelled and are mucoadhesive, but as non-porous and non-swellable materials opportunities for their drug loading were limited [19].

The ability of thiolated nanoparticles to adhere to, and to retain on different mucosal tissues has been investigated in vitro [5, 20-22]. Mucoadhesion and retention on mucosal surfaces is due to disulphide bond formation between thiol groups available in mucin glycoproteins and on the surface of the nanoparticles [23]. It was reported that these materials exhibited strong adherence to ocular mucosal surfaces, but PEGylation of the nanoparticles significantly reduced mucoadhesion due to the reduction in the number of free thiol groups, but improved mucus penetration $[5,20,21]$. As the cornea acts as a chemical and mechanical barrier to the passage of external materials, including drugs, penetration of thiolated and PEGylated (750 Da, $5000 \mathrm{Da}$ ) 
nanoparticles was explored on intact, pre-treated (with $\beta$-cyclodextrin, a penetration enhancer) and scraped epithelium tissues of the cornea. Fluorescence microscopy images showed that neither thiolated nor PEGylated nanoparticles penetrated the cornea or epithelium treated with $\beta$ cyclodextrin; when the epithelium was removed only PEGylated (5000 Da) nanoparticles permeated into the stroma [21]. Additionally, mucoadhesion of thiolated and PEGylated (750 and $5000 \mathrm{Da}$ ) nanoparticles on porcine urinary bladder mucosa was compared to nonmucoadhesive dextran (negative control) and the well-established mucoadhesive material chitosan (positive control). Following sequential washing with artificial urine, the thiolated nanoparticles were retained on the bladder mucosa for longer periods than dextran but for shorter time than chitosan. Again, PEGylation significantly reduced mucoadhesion which was correlated with reduced thiol content. Another study used various poly(2-alkyl-2-oxazoline)s to functionalise thiolated nanoparticles and penetration of the POZylated nanoparticles with different side chain length (methyl, ethyl and propyl) into gastric mucosa was assessed using fluorescence microscopy. Penetration significantly reduced as the alkyl chain length increased and POZylation reduced nanoparticles mucoadhesion due to a reduction of their surface thiol groups content [20].

Here, thiolated organosilica nanoparticles were synthesized following the protocol introduced by Irmukhametova et al., with modifications [5]. Particles were produced by self-condensation of MPTS in various solvents (predominantly DMSO) with or without bubbling with atmospheric or enriched oxygen. Reaction conditions also varied monomer concentration, catalyst choice and concentration and reaction temperature. The resulting nanoparticles were characterised using dynamic light scattering, transmission electron microscopy and zeta potential measurements. By systematically exploring reaction conditions, relationship between resultant particle size and the 
dielectric constant of the solvent or catalyst concentrations were defined allowing rational design of thiolated organosilica nanoparticle with required sizes.

\section{EXPERIMENTAL SECTION:}

Materials: 3-Mercaptopropyltrimethoxysilane (95\%), N-methyl-2-pyrrolidine (NMP), acetonitrile $(\mathrm{AcN})$, trimethylamine (TEA, $\geq 99 \%$ ), diethylamine (DEA, $\geq 99.5 \%$ ), sodium phosphate dibasic ( $\geq 99 \%)$, sodium phosphate monobasic dihydrate $(\geq 99 \%), 5,5^{\prime}$-dithiobis $(2$ nitrobenzoic acid) (DTNB, $\geq 98 \%$ TLC) and acetone were purchased from Sigma-Aldrich (UK). Dimethyl sulfoxide (DMSO), dimethyl formamide (DMF), tetrahydrofuran (THF), and sodium hydroxide pellets were purchased from Fisher Scientific (UK). 1, 4-dioxane (dioxane) was purchased from Acros (UK). Hydrochloric acid solution 1mol/L was from Fluka (UK). Lcysteine hydrochloride anhydrous (98\%) was purchased from Alfa Aesar (UK). All materials were used as received.

Synthesis of thiolated nanoparticles. Nine different synthetic conditions were used to prepare organosilica nanoparticles:

Effect of catalyst:

(1) MPTS $(0.75 \mathrm{~mL} ; 0.2 \mathrm{~mol} / \mathrm{L})$ was mixed with DMSO $(20 \mathrm{~mL})$ and $0.5 \mathrm{~mL}$ of serial concentrations of $\mathrm{NaOH}(0.05 ; 0.1 ; 0.2 ; 0.3 ; 0.5 ; 0.7$ and $0.9 \mathrm{~mol} / \mathrm{L})$. The reaction mixtures were left for 24 hours at room temperature with air bubbling and continuous stirring.

(2) MPTS (0.75 mL; $0.2 \mathrm{~mol} / \mathrm{L})$ was mixed with DMSO $(20 \mathrm{~mL})$ and $0.5 \mathrm{~mL}$ of serial concentrations of $\mathrm{HCl}(0.1 ; 0.3 ; 0.5 \mathrm{~mol} / \mathrm{L})$. The reaction mixtures were left for 24 hours at room temperature with air bubbling and continuous stirring. 
(3) MPTS (0.75 mL; $0.2 \mathrm{~mol} / \mathrm{L})$ was mixed with DMSO $(20 \mathrm{~mL})$ and $0.5 \mathrm{~mL}$ of diethylamine

$(0.2 \mathrm{~mol} / \mathrm{L})$ and left for 24 hours at room temperature with air bubbling and continuous stirring.

(4) MPTS (0.75 mL; $0.2 \mathrm{~mol} / \mathrm{L})$ was mixed with DMSO $(20 \mathrm{~mL})$ and $0.5 \mathrm{~mL}$ of triethylamine

$(0.2 \mathrm{~mol} / \mathrm{L})$ and left for 24 hours at room temperature with air bubbling and continuous stirring.

\section{Effect of monomer concentration:}

(5) Serial volumes of MPTS $(0.15 ; 0.25 ; 0.5 ; 0.75 ; 1.0$ and $1.5 \mathrm{~mL} ; 0.04 ; 0.07 ; 0.13 ; 0.2 ; 0.27$

and $0.4 \mathrm{~mol} / \mathrm{L})$ were mixed with DMSO $(20 \mathrm{~mL})$ and $0.5 \mathrm{~mL}$ of $0.5 \mathrm{~mol} / \mathrm{L} \mathrm{NaOH}$. The reaction mixtures were left for 24 hours at room temperature with air bubbling and continuous stirring.

\section{Effect of solvent:}

(6) MPTS $(0.75 \mathrm{~mL}$ or $0.38 \mathrm{~mL} ; 0.2 \mathrm{~mol} / \mathrm{L}$ or $0.1 \mathrm{~mol} / \mathrm{L}$ ) was mixed with organic solvent (20 $\mathrm{mL})$ and $0.5 \mathrm{~mL}$ of $\mathrm{NaOH}(0.5 \mathrm{~mol} / \mathrm{L})$ and left for 24 hours at room temperature with air bubbling and continuous stirring. The following organic solvents were used: DMF, NMP, AcN, THF, dioxane and acetone.

Effect of temperature:

(7) MPTS (0.75 mL; $0.2 \mathrm{~mol} / \mathrm{L})$ was mixed with DMSO $(20 \mathrm{~mL})$ and $0.5 \mathrm{~mL}$ of $\mathrm{NaOH}(0.5$ $\mathrm{mol} / \mathrm{L}$ ) and left for 24 hours at $50{ }^{\circ} \mathrm{C}$ with air bubbling and continuous stirring.

\section{Effect of air bubbling:}

(8) MPTS (0.75 mL; $0.2 \mathrm{~mol} / \mathrm{L})$ was mixed with DMSO $(20 \mathrm{~mL})$ and $0.5 \mathrm{~mL}$ of serial concentrations of $\mathrm{NaOH}(0.05 ; 0.1 ; 0.2 ; 0.3 ; 0.5 ; 0.7$ and $0.9 \mathrm{~mol} / \mathrm{L})$. The reaction mixtures were left for 24 hours at room temperature with continuous stirring but no air bubbling. 
(9) MPTS (0.75 mL; $0.2 \mathrm{~mol} / \mathrm{L})$ was mixed with DMSO $(20 \mathrm{~mL})$ and $0.5 \mathrm{~mL}$ of $\mathrm{NaOH}(0.5$ $\mathrm{mol} / \mathrm{L})$ and left for 24 hours at room temperature with enriched oxygen $\left(95 \% \mathrm{O}_{2}: 5 \% \mathrm{CO}_{2}\right)$ bubbling and continuous stirring.

All synthesized nanoparticles were purified by dialysis against deionized water ( 5 L, eight changes of water) using dialysis tubing with a 12000-14000 Da molecular weight cut off (Medicell Membranes Ltd., UK). The purified aqueous dispersions of the nanoparticles were stored at $4{ }^{\circ} \mathrm{C}$.

Dynamic Light Scattering and Zeta-Potential Measurements. Dynamic light scattering and zeta-potential measurements were conducted with dilute dispersions of nanoparticles at $25{ }^{\circ} \mathrm{C}$ using a Nano-S Zetasizer (Malvern Instruments, UK). Each batch of nanoparticles was synthesized in triplicate and the analysis was carried out three times for each sample. The mean and standard deviation of particle size, polydispersity and zeta-potential were calculated.

Transmission Electron Microscopy (TEM). TEM images of thiolated nanoparticles were acquired using a JEOL 2010 TEM operating at an accelerating voltage of $200 \mathrm{kV}$. For sample preparation, carbon coated $\mathrm{Cu}$ grids were brought into contact with the aqueous nanoparticle dispersions for $60 \mathrm{~s}$. One sample from each $\mathrm{NaOH}$ catalyst concentration was analysed and ImageJ software was used to process the images for size measurements. The mean \pm standard deviation values of nine measurements were calculated for each sample.

Ellman's Assay. The thiol group content in nanoparticles was determined by Ellman's assay. Before analysis, all nanoparticles were freeze-dried using a Heto Power Dry LL 3000 freezedrier (Thermo Electron Corporation). The dispersions $(0.3 \mathrm{mg} / \mathrm{mL})$ of nanoparticles were prepared in $10 \mathrm{~mL}$ phosphate buffer solution $(0.5 \mathrm{~mol} / \mathrm{L}, \mathrm{pH} 8)$ and were allowed to hydrate for 1 
h. In the meantime, $3 \mathrm{mg}$ of the Ellman's reagent (DTNB) was dissolved in $10 \mathrm{~mL}$ of $0.5 \mathrm{~mol} / \mathrm{L}$ phosphate buffer solution at $\mathrm{pH} 8$. After particle hydration, $500 \mu \mathrm{L}$ of DTNB solution was added to $500 \mu \mathrm{L}$ aliquots of the nanoparticle dispersion and incubated in the dark for $90 \mathrm{~min}$. The nanoparticle dispersion was then centrifuged for $10 \mathrm{~min}$ at 13,000 rpm (Sanyo, MSE Micro Centaur) and $200 \mu \mathrm{L}$ of the supernatant was placed in a 96-well microtiter plate. Absorbance was measured at $420 \mathrm{~nm}$ with a plate reader (Epoch, BioTek). Thiol concentration was calculated from a calibration curve of cysteine hydrochloride prepared as a series of solutions under the same conditions from $0.004-0.634 \mu \mathrm{mol} / \mathrm{mL}$ (Figure S1). The analysis was carried out three times and the mean \pm standard deviation values were calculated.

\section{RESULTS AND DISCUSSION:}

Synthesis of thiolated silica nanoparticles: Thiolated silica nanoparticles were synthesized following the protocol introduced by Irmukhametova et al. [5], using MPTS as the monomer. The hydrolysis and condensation of the monomer and the proposed structure of MPTS nanoparticles is presented in Scheme 1. As in the protocol, $\mathrm{NaOH}$ was the principle catalyst, though others were evaluated as below, and DMSO was the typical solvent [5] but, again, others were explored. The literature protocol employed air bubbling through the reaction media and again was used here, alongside no air bubbling and bubbling with $95 \% \mathrm{O}_{2}$. 
<smiles>CO[Si](C)(CCCS)O[Si](O)(CCSCCC[Si](O)(O)O[Hg]CCC[Si](O)(O)O[Si](O)(O)CCC[Hg])OC</smiles>
(MPTS)

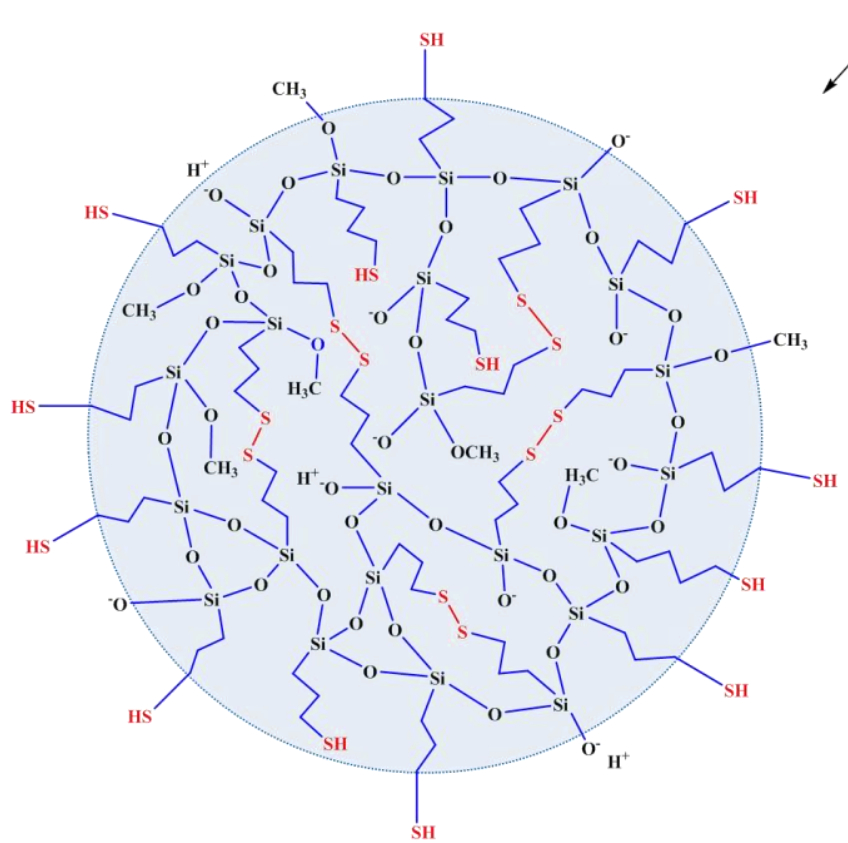

Scheme 1: Three main stages of nanoparticle formation and the proposed structure of MPTS nanoparticles.

Effect of catalyst: It was anticipated that, for a defined concentration of MPTS, in a given solvent at a fixed temperature and with air bubbling, increasing the catalyst concentration would decrease the resultant particle size; the results from this study, using $\mathrm{NaOH}$ as the catalyst, largely confirmed this expectation as shown in Table 1. 
Table 1: Characterization of thiolated organosilica nanoparticles prepared in DMSO using $0.2 \mathrm{~mol} / \mathrm{L}$ MPTS with varying concentrations of $\mathrm{NaOH}$ under continuous stirring and air bubbling.

\begin{tabular}{ccccccc}
\hline $\begin{array}{c}\text { Concentration of NaOH, } \\
\mathrm{mol} / \mathrm{L}\end{array}$ & $\begin{array}{c}\text { Size (DLS), } \\
\mathrm{nm}\end{array}$ & PDI & $\begin{array}{c}\text { Size (TEM), } \\
\mathrm{nm}\end{array}$ & $\begin{array}{c}\xi \text {-Potential, } \\
\mathrm{mV}\end{array}$ & $\begin{array}{c}\text { Yield, } \\
\mathrm{w} / \mathrm{w} \%\end{array}$ & $\begin{array}{c}\text { SH content, } \\
\mu \mathrm{mol} / \mathrm{g}\end{array}$ \\
\hline 0.05 & $291 \pm 30$ & $0.151 \pm 0.017$ & $586 \pm 119$ & $-25 \pm 8$ & $2 \pm 1$ & $127 \pm 52$ \\
0.1 & $193 \pm 22$ & $0.114 \pm 0.014$ & $100 \pm 29$ & $-40 \pm 2$ & $8 \pm 12$ & $121 \pm 54$ \\
0.2 & $161 \pm 40$ & $0.162 \pm 0.047$ & $78 \pm 21$ & $-32 \pm 4$ & $24 \pm 15$ & $131 \pm 49$ \\
0.3 & $59 \pm 3$ & $0.090 \pm 0.003$ & $35 \pm 5$ & $-45 \pm 2$ & $40 \pm 2$ & $150 \pm 39$ \\
0.5 & $50 \pm 1$ & $0.134 \pm 0.010$ & $27 \pm 3$ & $-55 \pm 2$ & $49 \pm 31$ & $152 \pm 55$ \\
0.7 & $47 \pm 2$ & $0.162 \pm 0.009$ & $22 \pm 4$ & $-34 \pm 1$ & $55 \pm 28$ & $152 \pm 19$ \\
0.9 & $47 \pm 5$ & $0.169 \pm 0.040$ & $16 \pm 3$ & $-35 \pm 12$ & $53 \pm 15$ & $113 \pm 15$ \\
\hline
\end{tabular}

Data from both DLS and TEM (Figure 1 and S2) showed that particle size decreases with increasing concentration of $\mathrm{NaOH}$, but to a lower limit. From the DLS analysis, relatively large particles were synthesized using our most dilute 0.05 and $0.1 \mathrm{~mol} / \mathrm{L} \mathrm{NaOH}$ with sizes of $\sim 290$ and $\sim 195 \mathrm{~nm}$, respectively. Using $\geq 0.5$ up to $0.9 \mathrm{~mol} / \mathrm{L} \mathrm{NaOH}$ did not further reduce particle size resulting in the nanoparticles of $\sim 50 \mathrm{~nm}$ (Table 1). Similarly, different concentrations of $\mathrm{NaOH}$ (1-5 mmol/L) were used for the synthesis of silica nanoparticles from TEOS; higher concentrations resulted in the formation of smaller and more uniform particles [24]. Particle size was previously reported to decrease when $2 \%$ and $27 \% \mathrm{NH}_{4} \mathrm{OH}$ catalyzed the synthesis of thiolorganosilica nanoparticles [2].

A similar trend but with differences in values was seen in the TEM data for the same particles. Unlike DLS, TEM measures the size of the particle per se rather than the particle with its hydration layer and so smaller particle sizes are typically recorded by electron microscopy. This is evident for the particles produced with 0.1 to $0.7 \mathrm{~mol} / \mathrm{L} \mathrm{NaOH}$ where the "core" particle size decreases to a lower limit of $\sim 35 \mathrm{~nm}$ and all sizes are somewhat smaller than the DLS values. 
However, TEM shows that very large particles are produced with the most dilute catalyst $(0.05$ $\mathrm{mol} / \mathrm{L}$, particles $\sim 586 \mathrm{~nm}$ ). The images (Figure S2) suggest that these particles are less dense, and may result from aggregation of primary particles or poor and/or incomplete synthesis noting that the yield for this reaction was very low (2\%). Interestingly, following an apparent plateau in size with 0.3 to $0.7 \mathrm{~mol} / \mathrm{L} \mathrm{NaOH}$, further increase in the catalyst concentration to 0.9 mol/L appears to further reduce the particle size as determined by TEM $(\mathrm{p}<0.05)$.

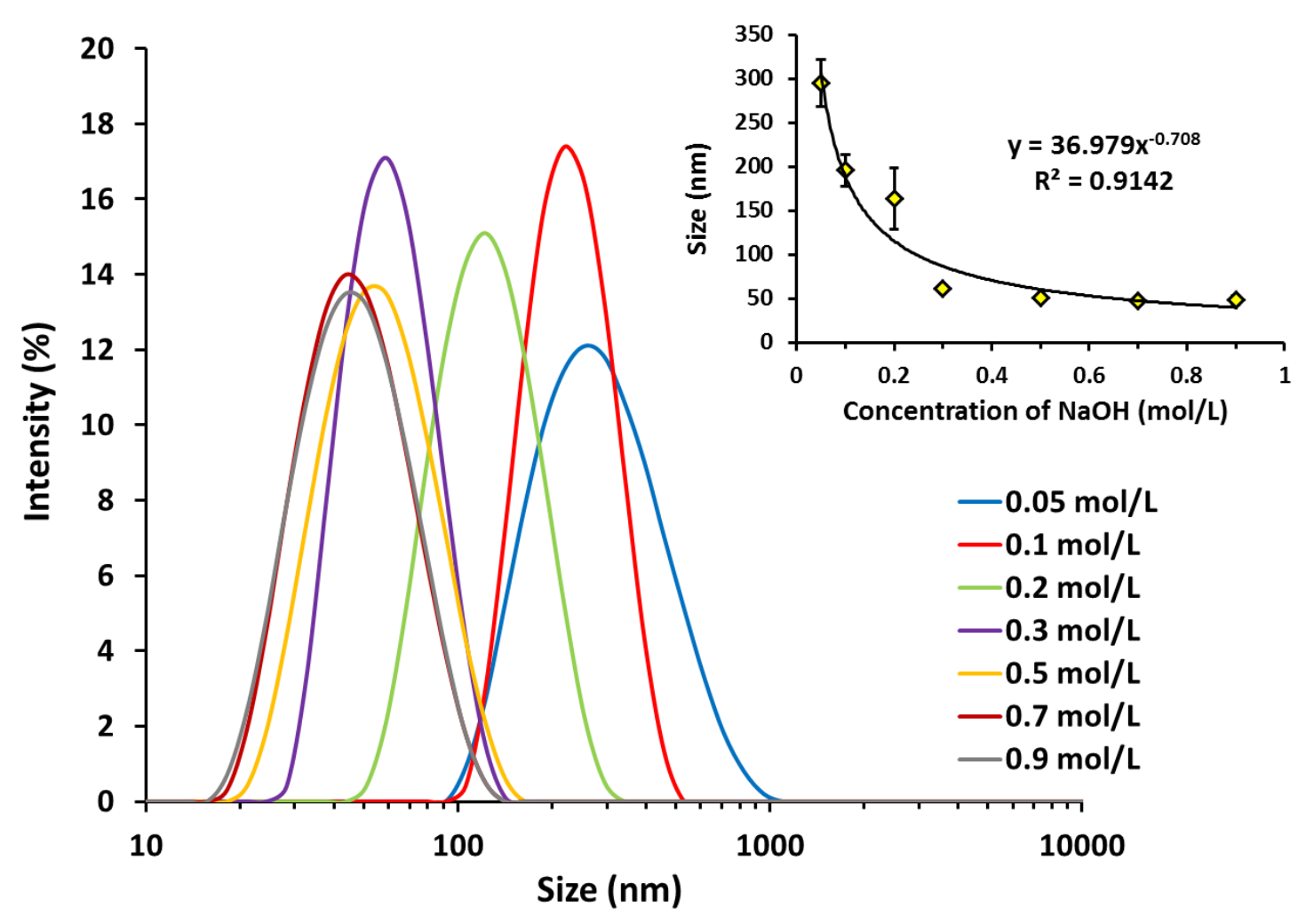

Figure 1: Dynamic light scattering size distributions of particles prepared using different concentrations of $\mathrm{NaOH}$ catalyst with $0.2 \mathrm{~mol} / \mathrm{L}$ MPTS in DMSO. Insert: The relationship between mean ( \pm SD) DLS size of thiolated organosilica nanoparticles and the concentration of $\mathrm{NaOH}$ used in the reaction mixture.

All the nanoparticles carry a net negative zeta-potential, attributed to the presence of $\mathrm{Si}^{-} \mathrm{O}^{-}$and $\mathrm{S}^{-}$groups at their surfaces. No clear trend was seen between the zeta-potential and catalyst concentration. 
The thiol content on the surface of the nanoparticles was determined using Ellman's assay and ranged from 113 to $152 \mu \mathrm{mol} / \mathrm{g}$, with a general trend of increasing content per gram with decreasing particle size (and increasing catalyst concentration). This could be due to the availability of larger number of $-\mathrm{SH}$ groups able to react with the Ellman's reagent arising from the increase in surface area per gram of the smaller particles. The yields of nanoparticles prepared using low concentration of $\mathrm{NaOH}$ were relatively poor $(2-24 \%)$, with higher yield recorded when $[\mathrm{NaOH}]>0.2 \mathrm{~mol} / \mathrm{L}$.

Alternative catalysts were investigated in the reaction, namely, $\mathrm{HCl}$, DEA and TEA, using the same protocol (Table S1). Particles obtained using different concentrations of $\mathrm{HCl}(0.1,0.3$ and $0.5 \mathrm{~mol} / \mathrm{L})$ were large $(1184,5309$ and $10,460 \mathrm{~nm}$ respectively) with negative zeta-potential and high polydispersity. In contrast, the bases DEA and TEA at $0.2 \mathrm{~mol} / \mathrm{L}$ produced particles in the same size range $(\sim 250 \mathrm{~nm})$, but statistically significantly larger $(\mathrm{P}<0.05)$ than those obtained using $\mathrm{NaOH}$. A reduction in particle size has been reported when the concentration of TEA was reduced in the reaction with TEOS $[11,13,14]$. In contrast, Shi et al. found that adding more TEA formed smaller particles [25]. This discrepancy may be due to other variations in the reaction conditions including using different monomer and solvent. It was reported that base catalysed condensation-mediated synthesis is faster than acid catalysed synthesis as the latter proceeds through hydrolysis and requires an additional step of condensation for nucleation which results in the formation of larger particles [26, 27]. Additionally, when $\mathrm{NaOH}$ was used instead of $\mathrm{NH}_{4} \mathrm{OH}$ as a catalyst in a 1:1 ratio of water and ethanol for synthesising nanoparticles from TEOS, reaction time reduced 60-fold and the obtained particles had a greater surface area compared to the particles produced using the conventional Stöber method [26]. 
Effect of monomer concentration: It was anticipated that, at a fixed catalyst concentration, increasing the monomer concentration would increase the particle size. Whilst, particle size was modified by varying the concentration of monomer (Table 2), for very dilute starting monomer concentrations $(0.04$ and $0.07 \mathrm{~mol} / \mathrm{L})$ an initial decrease in size with increasing monomer concentration was seen. However, from the DLS size distribution of the lowest concentration (Figure S3), there were two principle particle populations with the majority $\geq 100 \mathrm{~nm}$ and fewer that were 10- $20 \mathrm{~nm}$ resulting in a larger mean particle size with wide SD values. As monomer concentration increased, the proportion of smaller to larger particles increased. This could be due nucleation being restricted at lower concentrations, adsorption of oligomer nuclei on the surface of existing nuclei or the fusion of several silica nuclei which results in the formation of larger particles [28-30]. Nucleation and growth of nanoparticles from different materials in solutions was reviewed and is highly dependent on the reaction conditions; relatively minor changes may result in different particle growth mechanism [31]. It was reported that particles prepared using the Stöber method grow by nucleation followed by diffusion controlled coalescence [31, 32]. Subsequently, it was reported that particle growth by the Stöber method was by continuous nucleation of primary particles simultaneously with their aggregation [31, 33]. Here, with monomer concentrations $\geq 0.13 \mathrm{~mol} / \mathrm{L}$, particle size increased with increasing monomer concentration. There was a significant difference in size between the larger particles obtained using both a very low concentration of MPTS $(0.04 \mathrm{~mol} / \mathrm{L}, \mathrm{P}<0.05)$ and ten times that concentration $(0.4 \mathrm{~mol} / \mathrm{L}, \mathrm{P}<0.01)$ when compared to particles prepared using our "standard" $0.2 \mathrm{~mol} / \mathrm{L} \mathrm{MPTS}$ or indeed for MPTS concentrations between the extremes (Figure 2). All nanoparticles had negative zeta-potentials $(-14$ to $-45 \mathrm{mV})$ with relatively low yields $(18-50 \%)$ and wide polydispersities (0.640-0.360) were seen at lower MPTS concentrations. 
Table 2: Characterization of thiolated organosilica nanoparticles prepared in DMSO using $0.5 \mathrm{~mol} / \mathrm{L} \mathrm{NaOH}$ and by varying the concentrations of MPTS with continuous stirring and air bubbling.

\begin{tabular}{ccccc}
\hline $\begin{array}{c}\text { Concentration of MPTS, } \\
\mathrm{mol} / \mathrm{L}\end{array}$ & $\begin{array}{c}\text { Size (DLS), } \\
\mathrm{nm}\end{array}$ & PDI & $\begin{array}{c}\xi \text {-Potential, } \\
\mathrm{mV}\end{array}$ & $\begin{array}{c}\text { Yield, } \\
\mathrm{w} / \mathrm{w} \%\end{array}$ \\
\hline 0.04 & $113 \pm 22$ & $0.620 \pm 0.031$ & $-41 \pm 2$ & $40 \pm 10$ \\
0.07 & $51 \pm 30$ & $0.638 \pm 0.248$ & $-42 \pm 2$ & $53 \pm 1$ \\
0.13 & $38 \pm 1$ & $0.361 \pm 0.053$ & $-46 \pm 5$ & $24 \pm 4$ \\
$0.2 *$ & $50 \pm 1$ & $0.134 \pm 0.010$ & $-55 \pm 2$ & $49 \pm 31$ \\
0.27 & $63 \pm 2$ & $0.154 \pm 0.015$ & $-35 \pm 30$ & $48 \pm 10$ \\
0.40 & $120 \pm 16$ & $0.102 \pm 0.025$ & $-13 \pm 23$ & $18 \pm 6$ \\
\hline
\end{tabular}

* data for 0.2 mol/L MPTS taken from Table 1 .

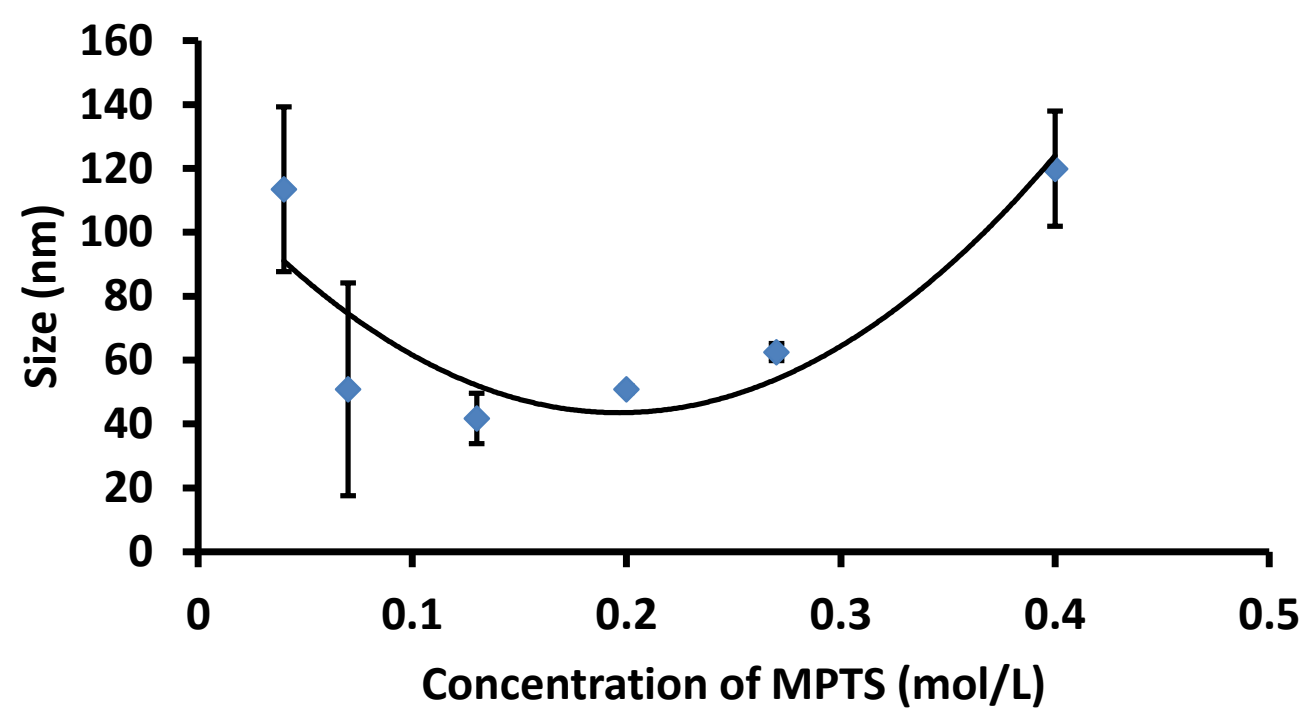

Figure 2: Mean \pm SD, dynamic light scattering sizes of particles prepared using various concentrations of MPTS compared to the standard protocol using $0.2 \mathrm{~mol} / \mathrm{L} \mathrm{MPTS,}(0.04$ $\mathrm{mol} / \mathrm{L}, \mathrm{P}<0.05,0.27 \mathrm{~mol} / \mathrm{L}, \mathrm{P}<0.01,0.4 \mathrm{~mol} / \mathrm{L}, \mathrm{P}<0.01)$

Nakamura et al. used different concentrations of (3-mercaptopropyl)trimethoxysilane, (3-

mercaptopropyl)triethoxysilane and (3-mercaptopropyl)methyldimethoxysilane to prepare silica nanoparticles, alongside tetraethoxysilane (TEOS). The nanoparticles were synthesized by the Stöber method using $65 \%$ ethanol with water and either $2 \%$ or $27 \%$ ammonium hydroxide. Their 
results showed that using $27 \% \mathrm{NH}_{4} \mathrm{OH}$ was optimal for thiol-organosilica nanoparticle synthesis when compared to TEOS and that particle size increased with increasing the concentration of the thiol-organosilica source. However, the time for complete formation of the nanoparticles varied (between 1 to 3 days), and was dependent on the synthetic conditions and concentration of the monomer and catalyst $[2,3]$. In our case, we report a similar increase in particle size with increasing monomer concentration, from $0.13 ; 0.2 ; 0.27$ to $0.4 \mathrm{~mol} / \mathrm{L}$ of MPTS in DMSO. However, our data also show that there is a lower limit to the size-related effects of the silica source concentration and that with very low concentrations of MPTS $(0.04 ; 0.07 \mathrm{~mol} / \mathrm{L})$, particle size decreases with increasing monomer concentration.

Effect of solvent: Our standard protocol synthesizes nanoparticles from dimethylsulfoxide [5]. Here, we additionally used various organic aprotic solvents with different dielectric constants to determine the influence on resultant particle sizes, charge and thiol content. Although aprotic solvents were reported to not "formally" take part in sol-gel reactions, they may influence the reaction kinetics by increasing the strength of nucleophiles or decreasing the strength of electrophiles. Additionally, aprotic solvents were reported to promote base-catalyzed condensation as they hydrogen bond to electrophilic protonated silanols [27]. The growth of the primary particles, their aggregation and the size of secondary particles are all dependent on the thermodynamic parameters of the system which control colloidal stability [34]. The general characteristics of the organosilica nanoparticles synthesized in different organic solvents, with other reaction conditions fixed, are in Table 3. 
Table 3: Characterization of organosilica nanoparticles synthesized in different organic solvents $(20 \mathrm{~mL}), 0.2 \mathrm{~mol} / \mathrm{L}$ MPTS and $0.5 \mathrm{~mL}$ of $0.5 \mathrm{~mol} / \mathrm{L} \mathrm{NaOH}$ with continuous stirring and air bubbling.

\begin{tabular}{ccccccc}
\hline Solvent & $\begin{array}{c}\text { Solvent's } \\
\text { dielectric } \\
\text { constant }\end{array}$ & $\begin{array}{c}\text { Size (DLS), } \\
\mathrm{nm}\end{array}$ & PDI & $\begin{array}{c}\xi \text {-Potential, } \\
\mathrm{mV}\end{array}$ & $\begin{array}{c}\text { Yield, } \\
\mathrm{w} / \mathrm{w} \%\end{array}$ & $\begin{array}{c}\text { SH content, } \\
\mu \mathrm{mol} / \mathrm{g}\end{array}$ \\
\hline DMSO & 47.00 & $45 \pm 3$ & 0.181 & $-55 \pm 7$ & $58.6 \pm 0.4$ & $249 \pm 30$ \\
DMF & 38.00 & $62 \pm 8$ & 0.513 & $-53 \pm 6$ & $27.1 \pm 2.6$ & $227 \pm 60$ \\
AcN & 37.00 & $306 \pm 28$ & 0.108 & $-60 \pm 13$ & $10.7 \pm 4.2$ & $68 \pm 16$ \\
NMP & 32.20 & $99 \pm 16$ & 0.552 & $-63 \pm 4$ & $22.0 \pm 1.5$ & $203 \pm 122$ \\
Acetone & 21.00 & $233 \pm 25$ & 0.120 & $-53 \pm 6$ & $3.0 \pm 1.5$ & $*$ \\
THF & 7.50 & $387 \pm 29$ & 0.219 & $-64 \pm 5$ & $5.6 \pm 1.5$ & $*$ \\
Dioxane & 2.25 & $548 \pm 86$ & 0.120 & $-64 \pm 13$ & $7.3 \pm 5.5$ & $*$ \\
\hline
\end{tabular}

* Ellman's assay was not performed due to very low yields.

With all seven solvents, the resultant particles gave invariant and, as expected, negative zeta potentials due to the presence of the negatively charged-Si- $\mathrm{O}^{-}$and $-\mathrm{S}^{-}$groups on their surface. Thiol content when synthesized from DMSO, DMF, and NMP were also invariant though preparing particles from acetonitrile gave lower thiol content; yields from other solvents were too low to allow analysis. It was reported that dielectric constant affects particle size at the nucleation stage; higher dielectric constants provide greater static repulsive force between the particles than the van der Waals attractive force, preventing gathering of the nuclei so resulting in smaller particle formation [35]. Our data accords with this and shows that particle size is strongly dependent on the dielectric constant of the solvent; the higher the dielectric constant, the smaller the particle size, as illustrated in Figure 3. 


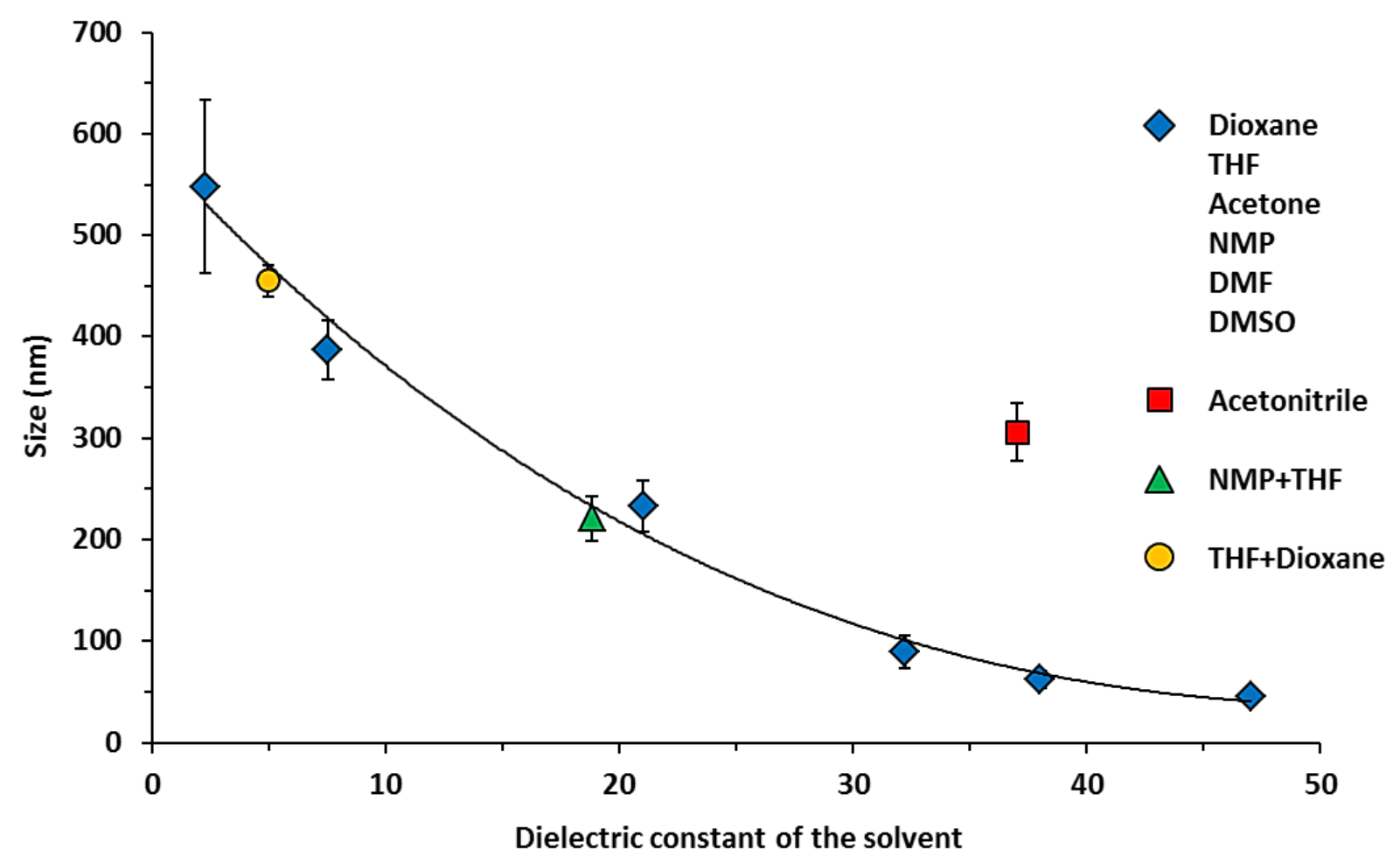

Figure 3: DLS size of organosilica nanoparticles synthesized in different organic solvents as a function of solvent's dielectric constant; solvents from left to right dioxane, THF, acetone, NMP, DMF, and DMSO; $y=-0.0015 x^{3}+0.3508 x^{2}-24.8 x+585.35 ; R^{2}=0.9894$

Further, the veracity of the relationship in Figure 3 was explored to determine if particle size could be rationally designed by selecting the dielectric constant of the aprotic solvent. Dielectric constants were selected by mixing NMP with THF and THF with dioxane, calculated by:

$$
d(x+y)=\frac{m * d(x)+n * d(y)}{m+n}
$$

where $d$ is a dielectric constant of a solvent; $x$ and $y$ are solvent 1 and 2 and $m$ and $n$ are the number of moles of solvents 1 and 2, respectively. The ratio of the solvents was $1: 1(\mathrm{v} / \mathrm{v})$ and the total volume was $20 \mathrm{~mL}$. The dielectric constant of 1:1 THF : dioxane was 4.94 which according to the relationship in Figure 3 was expected to provide particles of $471 \mathrm{~nm}$; experimental results provided material with a diameter of $455 \pm 16 \mathrm{~nm}$. The NMP : THF mixture, with a dielectric constant of 
18.8 was predicted to generate material of $233 \mathrm{~nm}$ with the experimental data in accord at $221 \pm$ $22 \mathrm{~nm}$. Further details can be found in Table S3.

As with thiol content, preparing particles from acetonitrile appears as an outlier and does not fit with the trend of smaller particles resulting from solvents with higher dielectric constants. Acetonitrile can be hydrolyzed in the presence of a strong aqueous base such as sodium hydroxide, essentially changing the dielectric constant of the solvent and, importantly, reducing the effective catalyst concentration; larger particles were formed with lower $\mathrm{NaOH}$ concentrations as shown in Table 1 [36].

In addition, AcN containing $0.1 \mathrm{~mol} / \mathrm{L}$ tetra-n-butylammonium perchlorate was shown to impact on molecular oxygen diffusivity and did not follow the Stokes-Einstein relationship due to the smaller size of molecular oxygen compared to the solvent molecules [37]. Moreover, AcN was reported to be a poor anion-solvating medium compared to DMSO [38]. Since oxygen is necessary for our nanoparticle production, then these findings may also partially explain the larger than predicted particle sizes from this solvent.

Von Storp et al. reported that using solvents with higher dielectric constants led to smaller protein nanoparticles. Whilst other processing conditions such as stirring rate and continuous addition of the desolating agent affected the human serum albumin nanoparticles, the authors showed that particle size could be predicted from the dielectric constant of the desolvation medium [39]. Whilst the mechanisms of nanoparticle production vary between our organosilica materials and proteinbased nanoparticles, both are determined by the solvent dielectric constant and generate materials with lower sizes in the region of $50 \mathrm{~nm}$. 
It has been reported that using an aprotic co-solvent formed spherical silica nanoparticles (Figure 4b) [13]. TEM images showed that our thiolated nanoparticles tend to self-assemble into chains and clusters due to the formation of disulfide bridges (Figure 4a).
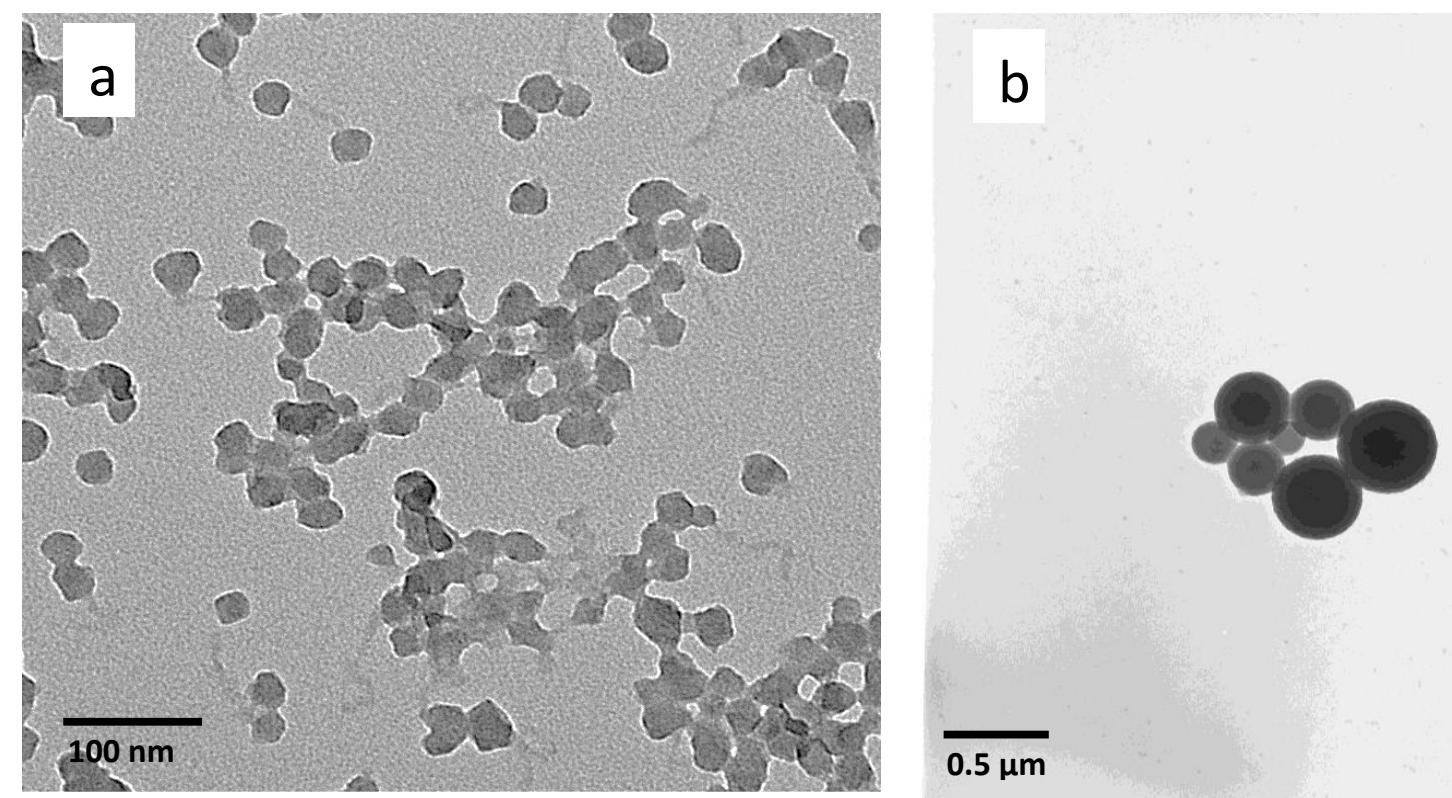

Figure 4: TEM images for organosilica nanoparticles synthesized in DMSO (a) and AcN (b)

Effect of temperature: Reaction temperature affects the size and dispersity of nanoparticles [34]. Synthesis was performed using our "standard" protocol (20 mL DMSO, $0.2 \mathrm{~mol} / \mathrm{L}$ MPTS and $0.5 \mathrm{~mL}$ of $0.5 \mathrm{~mol} / \mathrm{L} \mathrm{NaOH}$ with continuous stirring and air bubbling) but at $50{ }^{\circ} \mathrm{C}$ with the resultant particles significantly larger and more polydisperse (70 $\pm 1 \mathrm{~nm}$, PDI $0.188 \pm 0.029)$ than particles prepared at room temperature $(50 \pm 1 \mathrm{~nm}$, PDI $0.134 \pm 0.010)$. Similarly, Möller et al., using TEOS as silica precursor and TEA as the catalyst, reported a doubling in particle diameter when reaction temperature was raised from 60 to $80{ }^{\circ} \mathrm{C}[6,11]$. Nakamura et al. also reported the dependence of particle size on reaction temperature, alongside other factors such as monomer concentration as described above; larger particles were formed with higher concentrations of MPTS when temperature was increased [2,3]. However, a decrease in the size of particles produced by the Stöber method was reported as temperature increased to $60{ }^{\circ} \mathrm{C}$, 
beyond which temperature-size dependence was lost. This effect can be related to the production of a smaller number of nuclei at lower temperatures due to the change in hydrolysis rate. It was reported that varying the reaction temperature may change the viscosity and evaporation of ammonia or alcohol from the reaction mixture at elevated temperature $[34,40]$.

Effect of air bubbling: Our particles are typically prepared in the presence of atmospheric oxygen in order to facilitate oxidation of some thiol groups and formation of disulfide bridges. In addition, oxygen promotes the nucleation of primary particles and allows the particles to reach the stop-growth phase in shorter time; turbidity increased when the reaction mixture was bubbled with air, indicating the formation of colloidal particles [5]. Here, two batches of particles were prepared with serial concentrations of $\mathrm{NaOH}$ with and without air bubbling. Characterization of these particles is summarized in Table S2. When the reaction mixture was bubbled with air, smaller particles resulted with higher concentrations of the catalyst (i.e. $\geq 0.3$ $\mathrm{mol} / \mathrm{L}$ ) compared to lower catalyst concentrations, as described previously. Without air bubbling, when the catalyst concentration was $>0.3 \mathrm{~mol} / \mathrm{L}$ (shown above to generate small and invariant particle sizes- Table 1), larger particles tended to form in the absence of oxygen, shown in Figure 5. In addition to the role played by air bubbling in facilitating oxidation and formation of disulfide bridges, it may also provide more efficient stirring of reaction mixture. 


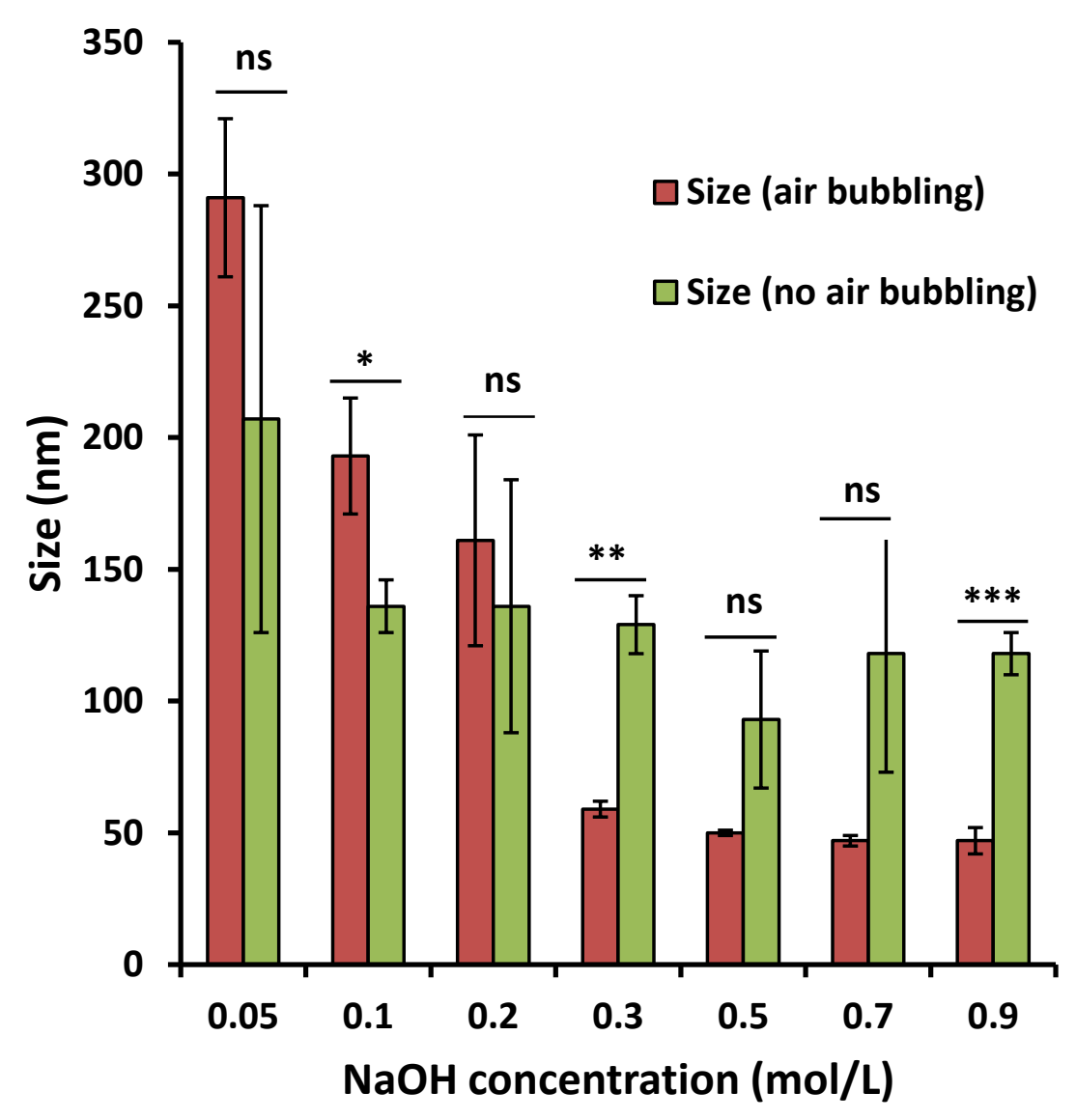

Figure 5: DLS size for particles prepared using serial concentrations of $\mathrm{NaOH}, 0.2 \mathrm{~mol} / \mathrm{L}$ MPTS, with continuous stirring, with and without air bubbling at room temperature.

Given the anomalous particles produced from acetonitrile discussed above and solvents effects on oxygen diffusivity, particles were prepared in acetonitrile in the presence and absence of air bubbling. Again, larger particles produced in the absence of oxygen $(416 \pm 13 \mathrm{~nm})$ compared to those formed in the presence of air bubbling (306 $\pm 28 \mathrm{~nm})$.

Further, since bubbling the reaction mixture with atmospheric air decreases particle size, bubbling with an oxygen enriched gas $(95 \%: 5 \%) \mathrm{O}_{2}: \mathrm{CO}_{2}$ was evaluated. Surprisingly, upon dialysis of the resultant product, a gel formed (Figure S3), probably from aggregation of the nanoparticles due to excessive formation of disulfide bridges. 


\section{CONCLUSION:}

The effects of synthetic conditions on the characteristics of thiol-organosilica particles were studied. Different catalysts produce particles with variable size ranges with smallest particles obtained when $\mathrm{NaOH}$ was selected. Increasing either the monomer concentration or reaction temperature increased the resultant particle size. Solvents with higher dielectric constants produced smaller particles and the experimental relationship was validated to demonstrate that specified particle sizes can be produced by manipulating the dielectric constant of the solvent with other conditions fixed. DMSO was selected as the base solvent as particles produced had the appropriate and consistent size, polydispersity and thiol content. Using this, bubbling the reaction mixture with atmospheric air promoted nucleation of primary particles and allowed the particles to reach the stop-growth phase in shorter time resulting in the formation of smaller

particles. Further enrichment of the reaction media with oxygen generated a gel due to excessive crosslinking. Our results demonstrate that, by selection and manipulation of reaction conditions including monomer concentration, catalyst type and concentration, reaction temperature and oxygenation, thiolated organosilica particles can be designed with a defined particle size. Studies into the internal structure of the nanoparticles synthesized under various synthetic conditions will be of interest in the future.

\section{SUPPORTING INFORMATION}

The Supporting Information is available free of charge on the ACS Publications website at xx. It includes calibration curve for Ellman's assay; TEM images for nanoparticles prepared by varying the concentration of $\mathrm{NaOH}$; dynamic light scattering distributions for particles prepared using 
0.04 and $0.07 \mathrm{~mol} / \mathrm{L}$ of MPTS, $0.5 \mathrm{~mol} / \mathrm{L} \mathrm{NaOH}$ and DMSO; tables with the values of sizes, zeta-potentials, yields and thiol content for particles prepared using different catalysts and different concentrations of $\mathrm{NaOH}$; characteristics of organic solvent mixtures and size (calculated vs. actual) of organosilica nanoparticles synthesized in those mixtures; images of gels formed when reaction mixture was bubbled with $95 \%: 5 \% \mathrm{O}_{2}: \mathrm{CO}_{2}$

\section{REFERENCES:}

(1) Nakamura, M.; Ishimura K. Synthesis and Characterization of Organosilica Nanoparticles Prepared from 3-Mercaptopropyltrimethoxysilane as the Single Silica Source. J. Phys. Chem. C. 2007. 111, 18892-18898.

(2) Nakamura, M.; Ishimura K. One-Pot Synthesis and Characterization of Three Kinds of Thiol-Organosilica Nanoparticles. Langmuir. 2008. 24, 5099-5108.

(3) Nakamura, M.; Ozaki S.; Abe M.; Doi H.; Matsumoto T.; Ishimura K. Size-controlled synthesis, surface functionalization, and biological applications of thiol-organosilica particles. Colloids Surf., B. 2010. 79, 19-26.

(4) Wang, L.; Zhao W.; Tan W. Bioconjugated silica nanoparticles: development and applications. Nano Research. 2008. 1.

(5) Irmukhametova, G.S.; Mun G.A.; Khutoryanskiy V.V. Thiolated Mucoadhesive and PEGylated Nonmucoadhesive Organosilica Nanoparticles from 3Mercaptopropyltrimethoxysilane. Langmuir. 2011. 27, 9551-9556.

(6) Tang, F.; Li L.; Chen D. Mesoporous Silica Nanoparticles: Synthesis, Biocompatibility and Drug Delivery. Adv. Mater. 2012. 24, 1504-1534.

(7) Schulz, A.; McDonagh C. Intracellular sensing and cell diagnostics using fluorescent silica nanoparticles. Soft Matter. 2012. 8, 2579-2585.

(8) Vivero-Escoto, J.L.; Huxford-Phillips R.C.; Lin W. Silica-based nanoprobes for biomedical imaging and theranostic applications. Chem. Soc. Rev. 2012. 41, 2673-2685.

(9) Rahman, I.A.; Padavettan V. Synthesis of silica nanoparticles by sol-gel: Size-dependent properties, surface modification, and applications in silica-polymer nanocomposites - a review. J. Nanomater. 2012. 2012, 15.

(10) Yamada, H.; Urata C.; Ujiie H.; Yamauchi Y.; Kuroda K. Preparation of aqueous colloidal mesostructured and mesoporous silica nanoparticles with controlled particle size in a very wide range from $20 \mathrm{~nm}$ to $700 \mathrm{~nm}$. Nanoscale. 2013. 5, 6145-53.

(11) Möller, K.; Kobler J.; Bein T. Colloidal Suspensions of Nanometer-Sized Mesoporous Silica. Adv. Funct. Mater. 2007. 17, 605-612. 
(12) Slowing, I.I.; Vivero-Escoto J.L.; Trewyn B.G.; Lin V.S.Y. Mesoporous silica nanoparticles: structural design and applications. J. Mater. Chem. 2010. 20, 7924-7937.

(13) Wu, S.-H.; Mou C.-Y.; Lin H.-P. Synthesis of mesoporous silica nanoparticles. Chem. Soc. Rev. 2013. 42, 3862-3875.

(14) Eisuke, Y.; Kazuyuki K. Colloidal Mesoporous Silica Nanoparticles. Bull. Chem. Soc. Jpn. 2016. 89, 501-539.

(15) Li, L.; Liang, R.; Li, Y.; Liu, H.; Feng, S. Hybrid thiol-ene network nanocomposites based on multi(meth)acrylate POSS. J. Colloid Interface Sci. 2013. 406, 30-36.

(16) Li, L.; Liu H. Rapid Preparation of Silsesquioxane- Based Ionic Liquids. Chemistry: A Eur. J. 2016. 22, 4713-4716.

(17) Nakamura, M.; Miyamoto K.; Hayashi K.; Awaad A.; Ochiai M.; Ishimura K. Time-lapse fluorescence imaging and quantitative single cell and endosomal analysis of peritoneal macrophages using fluorescent organosilica nanoparticles. Nanomedicine. 2013. 9, 27483.

(18) Irmukhametova, G.S.; Fraser B.J.; Keddie J.L.; Mun G.A.; Khutoryanskiy V.V. Hydrogen-bonding-driven self-assembly of PEGylated organosilica nanoparticles with poly(acrylic acid) in aqueous solutions and in layer-by-layer deposition at solid surfaces. Langmuir. 2012. 28, 299-306.

(19) Storha, A.; Mun E.A.; Khutoryanskiy V.V. Synthesis of thiolated and acrylated nanoparticles using thiol-ene click chemistry: towards novel mucoadhesive materials for drug delivery. RSC Advances. 2013. 3, 12275-12279.

(20) Mansfield, E.D.H.; de la Rosa V.R.; Kowalczyk R.M.; Grillo I.; Hoogenboom R.; Sillence K.; Hole P.; Williams A.C.; Khutoryanskiy V.V. Side chain variations radically alter the diffusion of poly(2-alkyl-2-oxazoline) functionalised nanoparticles through a mucosal barrier. Biomater. Sci. 2016. 4, 1318-1327.

(21) Mun, E.A.; Morrison P.W.; Williams A.C.; Khutoryanskiy V.V. On the barrier properties of the cornea: a microscopy study of the penetration of fluorescently labeled nanoparticles, polymers, and sodium fluorescein. Mol Pharm. 2014. 11, 3556-64.

(22) Mun, E.A.; Williams A.C.; Khutoryanskiy V.V. Adhesion of thiolated silica nanoparticles to urinary bladder mucosa: Effects of PEGylation, thiol content and particle size. Int. J. Pharm. 2016. 512, 32-38.

(23) Bernkop-Schnurch, A. Thiomers: a new generation of mucoadhesive polymers. Adv Drug Deliv Rev. 2005. 57, 1569-82.

(24) Dingsøyr, E.; Christy A.A. Effect of reaction variables on the formation of silica particles by hydrolysis of tetraethyl orthosilicate using sodium hydroxide as a basic catalyst. Prog. Colloid Polym. Sci. 2000. 116, 67-73.

(25) Chen, Y.; Chen H.-R.; Shi J.-L. Construction of Homogenous/Heterogeneous Hollow Mesoporous Silica Nanostructures by Silica-Etching Chemistry: Principles, Synthesis, and Applications. Acc. Chem. Res. 2014. 47, 125-137.

(26) Bhakta, S.; Dixit C.K.; Bist I.; Jalil K.A.; Suib S.L.; Rusling J.F. Sodium hydroxide catalyzed monodispersed high surface area silica nanoparticles. Mater Res Express. 2016. 3.

(27) C.Jeffrey Brinker, G.W.S., Hydrolysis and condensation II: Silicates, in Sol-gel science : the physics and chemistry of sol-gel processing. 1990, Academic Press Limited: London. p. 908. 
(28) Dixit, C.K.; Bhakta S.; Kumar A.; Suib S.L.; Rusling J.F. Fast nucleation for silica nanoparticle synthesis using a sol-gel method. Nanoscale. 2016. 8, 19662-19667.

(29) Ding, T.; Yao L.; Liu C. Kinetically-controlled synthesis of ultra-small silica nanoparticles and ultra-thin coatings. Nanoscale. 2016. 8, 4623-7.

(30) Carcouet, C.C.; van de Put M.W.; Mezari B.; Magusin P.C.; Laven J.; Bomans P.H.; Friedrich H.; Esteves A.C.; Sommerdijk N.A.; van Benthem R.A.; de With G. Nucleation and growth of monodisperse silica nanoparticles. Nano Lett. 2014. 14, 1433-8.

(31) Thanh, N.T.K.; Maclean N.; Mahiddine S. Mechanisms of Nucleation and Growth of Nanoparticles in Solution. Chem. Rev. 2014. 114, 7610-7630.

(32) Pontoni, D.; Narayanan T.; Rennie A.R. Time-Resolved SAXS Study of Nucleation and Growth of Silica Colloids. Langmuir. 2002. 18, 56-59.

(33) Green, D.L.; Lin J.S.; Lam Y.-F.; Hu M.Z.C.; Schaefer D.W.; Harris M.T. Size, volume fraction, and nucleation of Stober silica nanoparticles. J. Colloid Interface Sci. 2003. 266, 346-358.

(34) Plumeré, N.; Ruff A.; Speiser B.; Feldmann V.; Mayer H.A. Stöber silica particles as basis for redox modifications: Particle shape, size, polydispersity, and porosity. J. Colloid Interface Sci. 2012. 368, 208-219.

(35) Wang, H.-C.; Wu C.-Y.; Chung C.-C.; Lai M.-H.; Chung T.-W. Analysis of Parameters and Interaction between Parameters in Preparation of Uniform Silicon Dioxide Nanoparticles Using Response Surface Methodology. Ind. Eng. Chem. Res. 2006. 45, 8043-8048.

(36) Wang, Z.; Richter S.M.; Rozema M.J.; Schellinger A.; Smith K.; Napolitano J.G. Potential Safety Hazards Associated with Using Acetonitrile and a Strong Aqueous Base. Org. Process Res. Dev. 2017. 21, 1501-1508.

(37) Li, Q.; Batchelor-McAuley C.; Lawrence N.S.; Hartshorne R.S.; Compton R.G. Anomalous solubility of oxygen in acetonitrile/water mixture containing tetra-nbutylammonium perchlorate supporting electrolyte; the solubility and diffusion coefficient of oxygen in anhydrous acetonitrile and aqueous mixtures. J. Electroanal. Chem. 2013. 688, 328-335.

(38) Vasudevan, D.; Wendt H. Electroreduction of oxygen in aprotic media. J. Electroanal. Chem. 1995. 392, 69-74.

(39) von Storp, B.; Engel A.; Boeker A.; Ploeger M.; Langer K. Albumin nanoparticles with predictable size by desolvation procedure. J. Microencapsulation. 2012. 29, 138-146.

(40) Tan, C.G.; Bowen B.D.; Epstein N. Production of monodisperse colloidal silica spheres: Effect of temperature. J. Colloid Interface Sci. 1987. 118, 290-293. 